\title{
Efectos del programa affective e-learning en el desarrollo de la Competencia Digital en estudiantes del Grado en Educación Primaria
}

\section{Effects of the affective e-Learning program on the development of Digital Competence in Primary Education Degree students}

\author{
Álvaro Pérez García*1 \\ alvaro.perezgarcia@unir.net \\ Alba María Hernández-Sánchez** \\ albamaria@ugr.es \\ *Universidad Internacional de La Rioja (UNIR), Logroño, España \\ **Universidad de Granada, España
}

\section{Resumen:}

INTRODUCCIÓN. Este artículo presenta los efectos de un programa interuniversitario de especialización en competencias digitales basado en la metodología didáctica de affective e-learning. El programa formativo nace del proyecto I+D+i denominado Evaluación y desarrollo de dos competencias genéricas en estudiantes de primer año del grado de maestro en educación primaria. El grupo de 109 participantes pertenece al título de magisterio de Educación Primaria de las Facultades de Educación de la Universidad de Oviedo, la Universidad de Jaén y en la Universidad de Granada. MÉTODO. Tras el diseño del programa formativo se aplica un cuestionario ad-hoc validado por jueces expertos externos. El instrumento formado

\begin{abstract}
:
INTRODUCTION. This article presents the impact of an interuniversity specialization program about digital competences based on the Affective e-Learning methodology. The educational program originated from an $\mathrm{R}+\mathrm{D}$ project called Evaluation and development of two generic competences in first year Primary Education students. A sample of 109 students from the Primary Education Degree at the universities of Oviedo, Jaén and Granada participated in the study. METHOD. After completing the design of the educational program, an ad-hoc questionnaire validated by external experts was applied. The 49-item tool had a Cronbach Alpha value of 0.786 . This questionnaire was based on the Common Framework for Teaching Di-
\end{abstract}

1 Dirección para correspondencia (correspondence address):

Álvaro Pérez García. Universidad Internacional de La Rioja (UNIR), Logroño. C/ Tolosa Latour, 21. Bloque Interior, Planta menos 3, Puerta D. 11007-Cádiz (España). 
Efectos del programa affective e-learning en el desarrollo de la Competencia Digital en estudiantes del Grado en Educación Primaria

Álvaro Pérez García y Alba María Hernández-Sánchez

por 49 ítems tiene una estabilidad de escala aplicada con un Alfa de Cronbach de 0,786 . Este cuestionario se fundamenta en el Marco Común de Competencia Digital Docente del Instituto Nacional de Tecnologías Educativas y de Formación del Profesorado. RESULTADOS. Se demuestran diferencias significativas entre el pretest y el postest en la comprensión y aplicación de habilidades de la competencia digital, en términos de mejora en las destrezas de configuración, uso, autonomía, análisis, búsqueda y cumplimiento de estándares. DISCUSIÓN. Los hallazgos encontrados avalan la efectividad de aplicar un modelo de affective e-learning que se adapte a los conocimientos previos y los distintos ritmos y estilos de aprendizaje del grupo de participantes en su formación universitaria.

\section{Palabras clave:}

Affective e-learning; competencia digital; educación superior; evaluación de programas. gital Competence of the National Institute of Educational Technologies and Teacher Training. RESULTS. Significant differences were reported between pretests and posttests for the understanding and application of digital competence abilities, more specifically regarding configuration skills, use, autonomy, analysis, search and compliance to standards. DISCUSSION. Findings endorsed the effectivity of applying an affective e-learning model that adapts to students' preliminary knowledge and different learning paces and styles.

\section{Key words:}

Affective e-Learning; digital competence; higher education; program evaluation.

\section{Résumé:}

INTRODUCTION. Cet article présente les effets d'un programme interuniversitaire de spécialisation en compétences numériques basé sur la méthodologie didactique de l'elearning affectif. Le programme de formation est né du projet $R+D+i$ intitulé Evaluation et développement de deux compétences génériques chez les étudiants de première année du diplôme d'enseignant de l'enseignement primaire. Le groupe de 109 participants fait partie du diplôme universitaire de grado de l'enseignement primaire des facultés d'éducation de l'Université d'Oviedo, de l'Université de Jaén et de l'Université de Grenade. MÉTHODE. Après la conception du programme de formation, un questionnaire ad hoc validé par des juges experts externes est appliqué. L'instrument composé de 49 éléments a une stabilité d'échelle appliquée avec un alpha de Cronbach de 0,786. Ce questionnaire est basé sur le Cadre Commun de Compétence pour l'Enseignement Numérique de I'Institut National des Technologies Educatives et de la Formation des Enseignants. RÉSULTATS. Des différences significatives sont démontrées entre le pré-test et le post-test dans la compréhension et l'application des compétences numériques, en termes d'amélioration des compétences de configuration, d'utilisation, d'autonomie, d'analyse, de recherche et de conformité avec les normes. DISCUSSION. Les résultats appuient l'efficacité de I'application d'un modèle d'apprentissage affectif en ligne qui s'adapte aux connaissances antérieures et aux différents rythmes et styles d'apprentissage du groupe de participants dans leur formation universitaire.

\section{Mots clés:}

e-learning affectif; compétence numérique; enseignement supérieur; évaluation des programmes.

Fecha de recepción: 27-02-2020

Fecha de aceptación: 15-06-2020 


\section{Introducción}

A pesar de ser considerados como nativos digitales, diversos estudios muestran que el alumnado universitario no necesariamente llega con un nivel óptimo de competencia digital a la Universidad (Gisbert y Esteve, 2011; Molina Jaén, Pérez García y Antiñolo Piñar, 2012; Centeno Moreno y Cubo Delgado, 2013; Esteve Mon, 2015; Vázquez-Cano et al., 2017), lo que conlleva, necesariamente, la realización de programas formativos que trabajen una de las competencias más importantes en los profesionales del futuro.

En el caso de los estudiantes que se están formando para ser futuros docentes, sea cual sea el nivel educativo, esto cobra mayor importancia aún, ya que la escuela siempre ha sido un motor de cambio social y, por ende, se tiene que adaptar a los rápidos avances tecnológicos para poder aplicarlos a los nuevos modelos didácticos.

Teniendo en cuenta que la competencia digital dentro del currículo oficial de los grados de educación ha ganado terreno como competencia transversal y están desapareciendo las asignaturas concretas que trabajan las TIC aplicadas a la educación, es necesario establecer una serie de programas formativos durante el primer año de estudios universitarios que refuercen la competencia digital con la que este alumnado llega a la Universidad, teniendo presente sus conocimientos previos a nivel de conocimientos, procedimientos y actitudes.

Para ello, es necesario que la metodología que se adapte en todo momento a las necesidades del alumnado, en definitiva, que facilite y propicie el aprendizaje del alumnado basándose en la personalización, la flexibilidad y la empatía. El programa que aquí se presenta, denominado "Programa Interuniversitario de Especialización en Competencias Comunicativo-Digitales", se realiza en la modalidad semipresencial mediante un conjunto de unidades didácticas hipermedia diseñadas para tal fin siguiendo un modelo innovador e inclusivo de aprendizaje denominado affective eLearning (Hernández-Sánchez y Ortega-Maldonado, 2015). Sus autores subrayan la necesidad y oportunidad de personalizar los procesos de creación de conocimiento, destacando el papel de los flujos emocionales positivos en el grado de bienestar del alumnado. Este clima de bienestar previene estados de frustración que conducen a la desmotivación y al abandono.

Con todo, y tras la aplicación del programa formativo en cuestión, 
se muestran las carencias del alumnado universitario con respecto al desarrollo de la competencia digital y, cómo a través de la formación y la alfabetización tecnológica, se pueden conseguir grandes avances para que los futuros maestros y maestras trabajen las TIC con fines didácticos en el aula de Educación Primaria, y fortalecer así la competencia digital tanto del docente como del alumnado. El proceso de diseño, desarrollo y evaluación de la experiencia se enmarcada dentro del programa de ayudas estatales de I+D+i con el proyecto "Evaluación y desarrollo de dos competencias genéricas en estudiantes de primer año del grado de maestro en educación primaria", referencia EDU2015-70491-R.

\section{Marco empírico}

\section{Competencia Digital}

Los principales avances educativos y el actual auge de los modelos de formación se deben a la incorporación de las Tecnologías de la Información y la Comunicación, así como a la aplicación de elementos pedagógicos provenientes de aproximación sociocultural. En esta competencia también confluyen acciones de comunicación, trabajo en equipo e innovación.

"La escuela es reposicionada como responsable de un proceso de alfabetización que incluye ahora también el acceso a nuevos lenguajes y códigos, que potencian y estructuran nuevos modos de pensar y comunicar de nuestra sociedad" (Roldán y Sabulski, 2012, p. 12). Y en ese marco, la figura del docente como actor clave recibe presiones, demandas y expectativas respecto a los cambios que las TIC podrían producir en el ámbito educativo y en la sociedad en su conjunto.

Para Romero, Hernández y Ordóñez (2016, p. 34), "Ios docentes no son ajenos a esta competencia y, por ello, es importante que sepan desenvolverse con los nuevos instrumentos que pueden utilizar en la educación".

Por ello, "la formación y la actualización del profesorado, es el camino adecuado para conseguir la incorporación de los recursos tecnológicos al terreno educativo", como defienden Mayorga, Madrid y Núñez (2016, p. 214). En este sentido, las habilidades digitales, imprescindibles tanto en la vida cotidiana de cualquier ciudadano como en el desarrollo 
académico y profesional de todos los alumnos, han entrado a formar parte del conjunto de destrezas no terminales o específicas cuyo conocimiento deberá acreditar el alumnado en el momento de su graduación como futuros maestros (Espuny, González y Gisbert, 2010).

Así, en nuestro país, el INTEF (2017) desarrolló el Marco Común de Competencia Digital Docente, un marco de referencia para el diagnóstico y la mejora de las competencias digitales del profesorado, compuesto de 5 áreas competenciales y 21 competencias estructuradas en 6 niveles competenciales, que partía del DIGCOMP, un Marco de desarrollo y Comprensión de la Competencias Digital en Europa.

\section{Programa Interuniversitario de Especialización en Competencias Comunicativo-Digitales}

El "Programa Interuniversitario de Especialización en Competencias Comunicativo-Digitales", surge de la necesidad de dar respuesta a las debilidades encontradas tras la implementación de los cuestionarios sobre competencia digital y sobre competencia comunicativa, creados para conocer el nivel de alfabetización comunicativo-digital del alumnado de $1 .^{\circ}$ del Grado de Magisterio. El programa creado se realizó en la modalidad semipresencial mediante un conjunto de unidades didácticas hipermedia diseñadas para tal fin siguiendo un modelo innovador e inclusivo de aprendizaje denominado Affective eLearning (Hernández-Sánchez y Ortega-Maldonado, 2015).

La creación de las unidades didácticas por parte de profesorado experto, se realizó en primer lugar en formato Word y, posteriormente se convirtió en hipermedia para su traslado a la plataforma situada en el Campus Virtual Inclusivo del Grupo Internacional de Investigación Tecnología Educativa e Investigación Social, que colaboró en este proyecto.

El programa formativo se compone de 3 unidades que abarcan todos los ítems de los cuestionarios en los que los resultados muestran carencias, aunque en el caso de este trabajo solo se expondrán las unidades que hacen referencia a la competencia digital.

La unidad 1 se denomina "Mejorando nuestra competencia comunicativa para crear y difundir textos", y en ella se trabajan aspectos relativos a mejorar las capacidades técnicas para planear y redactar textos que, posteriormente, se puedan difundir por escrito, mediante vOz o combinando voz y gestos; aprender las propiedades que identifican 
Efectos del programa affective e-learning en el desarrollo de la Competencia Digital en estudiantes del Grado en Educación Primaria

Álvaro Pérez García y Alba María Hernández-Sánchez

la correcta escritura de un texto; aplicar las estrategias que permiten componer textos coherentes; identificar las características de un texto argumentativo, diferenciándolo de otros tipos de textos; conocer los derechos de autor a tener en cuenta al generar obras textuales, sonoras, fotográficas o audiovisuales; conocer la forma de citar a autores para respetar la propiedad intelectual de ideas, modelos, buenas prácticas y resultados de investigación; ser capaz de crear un texto argumentativo de naturaleza académica, adaptando el discurso a la finalidad deseada; y autoevaluar y corregir un texto escrito de carácter argumentativo de creación propia.

La unidad 2 trabaja la "Identidad digital y la huella digital en Inter-

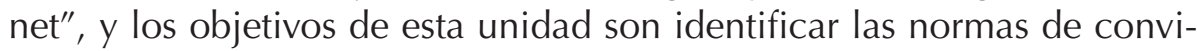
vencia adecuadas en las distintas aplicaciones de la Red, ser capaz de interactuar en un foro, en un chat o comunicarse por correo electrónico teniendo en cuenta las características socioculturales de las audiencias y respetando las normas de convivencia en la red, gestionar nuestro rastro digital de manera preventiva, ser capaz de configurar el navegador de nuestro ordenador para favorecer la privacidad de nuestra identidad y la seguridad de nuestros equipos y ser capaz de crear un video con un programa de edición sencillo.

La unidad 3 se denomina "Crea tu propio blog", y aquí se fomenta la utilización de los recursos digitales de forma didáctico-creativa. En este sentido, los principales objetivos son identificar las características principales de un blog en Internet; ser capaz de crear e interactuar en un espacio del tipo blog, produciendo uno y manteniendo comunicación escrita con otros posibles usuarios; y gestionar nuestro propio espacio Blog. Además, se relaciona esta unidad con las anteriores y así también el alumnado tiene que ser capaz de interactuar en un foro, en un chat o comunicarse teniendo en cuenta las características socioculturales de las audiencias y respetando las normas de convivencia en la red y gestionar nuestro rastro digital de manera preventiva.

Además, en la plataforma virtual se creó un repositorio de programas de libre uso relativos a grabación, edición, inclusión de texto, etc., además de tutoriales de uso para que el alumnado pueda dominar estos programas sin dificultad.

El programa formativo completo tuvo una duración de 2 meses y para obtener el certificado se debían realizar todas las actividades del mismo. 


\section{La competencia digital de los futuros maestros}

El cambio metodológico y de rol que ha experimentado el profesorado con la implantación de las TIC en el aula ha sido muy grande en los últimos años. Como exponen Cantón, Grande y Cañón (2016):

Los docentes se convierten en mediadores de los aprendizajes del alumnado para que éste logre aprendizajes significativos, docentes capaces de organizar el contexto en el que el alumnado ha de desarrollarse, potenciando su interacción con los materiales y con el trabajo colaborativo y compartiendo experiencias de aprendizaje con su alumnado, a la vez que atienden a las diferencias individuales de cada uno de ellos (p. 241).

La competencia digital en la formación inicial docente, exponen Girón-Escudero, Cózar-Gutiérrez \& González-Calero (2019, p. 193), “es esencial en la actualidad ya que los estudiantes de enseñanza básica están en frecuente contacto con las nuevas tecnologías. Por tanto, sería conveniente que los futuros docentes acabasen el grado con una competencia digital óptima".

En este sentido, los futuros maestros deben ser competentes en diversos aspectos relacionados con las TIC que incluyen una dimensión tecnológica, una dimensión pedagógica y una dimensión conceptual, es decir, el profesor que sabe utilizar técnicamente las TIC, el profesor que sabe utilizar las TIC como recurso didáctico y el profesor que domina los contenidos que va a enseñar a través de las TIC. En este caso, el programa formativo elaborado se centra en las dos primeras dimensiones, incidiendo en el conocimiento de recursos y herramientas TIC que el alumnado ha señalado que su dominio es escaso, e incidir en su uso pedagógico.

\section{Compartir información en la red}

Actualmente -expone Raro López (2012)- se han implementado nuevas herramientas de participación donde el lector está cada vez más presente en todas las fases del hipertexto, compartiendo conocimientos, participando en debates $y$, por tanto, adquiriendo un protagonismo impensable hace un par de décadas. En la misma línea Cáceres, Brändle y Ruiz (2017, p. 234) afirman que "son pocos los espacios sociales que no 
Efectos del programa affective e-learning en el desarrollo de la Competencia Digital en estudiantes del Grado en Educación Primaria

Álvaro Pérez García y Alba María Hernández-Sánchez

hayan sido transformados como resultado de las tecnologías digitales".

En este sentido, los futuros maestros deben formarse para que esa participación en la red sea adecuada y se pueda utilizar con fines didácticos. Argente, Vivancos, Alemany y García-Fornes (2017) señalan, pues, la importancia de que los estudiantes desarrollen habilidades para entender cómo unirse y construir estas redes, así como conocer las herramientas para hacerlo, su propósito, su intención, sus normas y sus protocolos.

Las redes sociales o la creación de blogs o webs, son recursos ya generalizados en los entornos personales de aprendizaje, pero el cómo se utilizan, los riesgos que conllevan o los lenguajes que se emplean, son poco trabajados en los planes de estudios de los distintos grados universitarios y, concretamente en los grados de Educación Infantil y Educación Primaria, es necesaria su inclusión en los procesos curriculares. Marín Díaz (2016) afirma así que la inclusión del blog en las aulas supone la modificación de las metodologías didácticas de los docentes.

Por ello, señala Serrano-Puche (2013) que:

La consolidación del medio online como un nuevo espacio, en el que la interacción y el elemento social cobran fuerza, origina que las relaciones mediadas por la tecnología puedan complementar y en ocasiones sustituir a la comunicación interpersonal cara a cara (p. 354).

En este sentido, es importante una adecuada interacción digital, como exponen Fernández Roldán y García García (2016), ya que:

Permitirá un mayor entendimiento y colaboración entre los miembros y actuantes de diversas generaciones y perfiles de usuarios, lo cual se reflejará en un mejor desempeño y, por ende, mayor satisfacción de a quienes se brinda un servicio, lo cual garantiza el buen trabajo realizado de los profesionales del área (p. 37).

Esta interacción digital también conlleva aspectos que, mirándolos de forma global, nos van a comprometer a cuidar nuestra imagen online, es decir, nuestra identidad digital, ya que este nuevo contexto sociocultural y educativo conlleva, como exponen Giones-Valls y Serrat-Brustenga (2010), que en la red coexisten una gran cantidad de datos personales, contribuyendo a crear nuevas identidades que pueden coincidir o no con la identidad analógica. Bermejo (2017, p. 38) define esta identidad 
como "el conjunto de aspectos, rasgos o elementos propios de una persona o individuo".

Por tanto, hay que valorar y controlar, otorgándole una capital importancia al nuevo concepto de identidad virtual como resultado de la interacción en las redes sociales y plataformas virtuales móviles ya que, como señala Portillo Fernández (2016):

A pesar de la temporalidad de la imagen en las redes y la ausencia de compromisos que señalan algunos expertos, la capacidad de poder diseñar una identidad eidética, inalterable, estática y seleccionada entre todas las características del sujeto, supone la evidente predilección frente a la personalidad real con todos sus rasgos (p. 60-61).

\section{La integración de lo audiovisual como recurso didáctico-creativo}

Tanto el alumnado como el profesorado están expuestos continuamente a la profusión de imágenes que nos dominan inevitablemente en la sociedad actual. Por ello, la inclusión de lo audiovisual en el currículo oficial en todos los niveles educativos es fundamental para formar a los futuros ciudadanos con una conciencia crítica hacia las imágenes.

Por ello, destaca García Sempere (2014, p.116) que, "con los nuevos avances tecnológicos y la alta presencia del vídeo en la vida de las personas, y en concreto en el ámbito escolar, aparecen nuevos desafíos para conseguir una educación más accesible y participativa".

En este sentido, y centrándonos en el nivel de Educación Primaria, el profesorado necesita que en los planes de estudio de su formación inicial se incluyan la creación, la crítica y el estudio histórico de los medios, como enlace con los nativos digitales que ya conviven desde que nace con los medios de comunicación.

Los medios audiovisuales en el aula poseen un doble uso, tal y como expone Rodríguez-López (2017, p. 165), como “instrumentos en sí mismos o como apoyo en el aprendizaje de otras asignaturas con la finalidad de hacerlas más atractivas para los alumnos y despertarles de la pasividad de los modelos tradicionales basados en las clases magistrales", fomentando proyectos que incluyan lo audiovisual.

Aunque Ezquerra, Burgos y Manso (2016) hablan de la complejidad de la creación audiovisual, desembocando, por tanto, en la necesidad de llevar a cabo una estructuración del proceso creativo en sucesivas 
Efectos del programa affective e-learning en el desarrollo de la Competencia Digital en estudiantes del Grado en Educación Primaria

Álvaro Pérez García y Alba María Hernández-Sánchez

etapas (selección de tópicos, guion literario, guion técnico...) y la consecuente obligación de trabajar en equipo de modo participativo y cooperativo, ello provoca que esta actividad sea un candidato idóneo para llevar a cabo una enseñanza por proyectos.

Así pues, en este trabajo se ha optado por la creación de proyectos audiovisuales como recurso didáctico, dado que es uno de los aspectos en el que se ha encontrado más carencias en el alumnado que acaba de llegar a la Universidad (vergüenza de visionarse o escucharse en una grabación, falta de formación en oratoria, argumentación o locución, escasez de medios o falta de práctica en el uso de los nuevos dispositivos...).

Trabajo como el de Torres Climent (2009) ya defendían que la grabación en vídeo de experiencias de laboratorio constituye un atractivo recurso para el aprendizaje de nuestros alumnos. Hidalgo y Aliaga (2020, p.80), exponen que, "la presencia de Recursos Audiovisuales en las instituciones escolares ha aumentado considerablemente, por los esfuerzos realizados desde los propios centros y puestos en funcionamiento por las diferentes Administraciones Educativas", aunque reflejan que "los criterios que se siguen manejando para su utilización, se apoyan más en principios administrativos, de costumbre, experienciales y operativos que en otros que pudiéramos denominar técnico -didácticos y curriculares" (p. 80).

León (2018) realizó en Perú una experiencia y un estudio con niños YouTubers, destacando que estos despliegan habilidades tanto técnicas como sociales y críticas desde la producción de contenido.

Otra interesante actividad para trabajar lo audiovisual en el aula es la creación de vídeos musicales. Rodríguez-López (2017) expone que:

En el contexto actual, cada vez más audiovisual, se erige como uno de los formatos más adecuados para el aprendizaje y la alfabetización en los medios gracias a su brevedad y condensación, su atractivo, su despliegue estético y técnico y su vinculación con la música (p. 166).

Ortega Carrillo y Pérez García (2013), elaboraron un "Programa de Alfabetización para el uso Didáctico del Cine Digital", que se implementó en alumnado de $3 .^{\circ}$ de la Diplomatura de Magisterio de Educación Infantil y Educación Primaria, obteniendo unos resultados muy interesantes en los futuros docentes. 
Hay, pues, algunas experiencias enriquecedoras que han trabajado lo audiovisual para mejorar la competencia digital, pero queda aún mucho camino por recorrer, pues lo ideal es que este tipo de programas se consolide en el currículo oficial de los planes de estudio de los grados de Educación Infantil y Educación Primaria de manera longitudinal.

\section{Desarrollo de la investigación}

\section{Objetivos}

El objetivo general de este trabajo es comprobar si el "Programa Interuniversitario de Especialización en Competencias Comunicativo-Digitales", realizado bajo la metodología de "affective e-Learning" (HernándezSánchez y Ortega-Maldonado, 2015), es adecuado para desarrollar competencias digitales en los futuros maestros de Educación Primaria.

Este objetivo general se puede descomponer en varios objetivos de carácter más específico:

a) Comprobar el dominio de las competencias digitales del alumnado universitario del grado de Educación Primaria.

b) Realizar una aproximación epistemológica a la competencia digital.

c) Crear un programa formativo interuniversitario, basado en el affective e-learning para desarrollar la competencia digital en los futuros docentes.

d) Exponer los resultados obtenidos del pretest y el postest; y extraer conclusiones y propuestas de futuro.

\section{Metodología}

Nos encontramos ante una investigación evaluativa que se concreta en la evaluación de un programa formativo, denominado "Programa Interuniversitario de Especialización en Competencias ComunicativoDigitales", en el que se pretende comprobar el nivel competencial del alumnado universitario que están realizando el grado de Educación Primaria en la Universidad de Oviedo, Universidad de Jaén y la Universidad de Granada con respecto a la competencia comunicativa y la competencia digital, y cómo la formación en el ámbito audiovisual puede 
favorecer la mejora de ambas competencias. Este artículo se va a centrar concretamente en la evaluación pretest-postest de la competencia digital de 109 alumnos y alumnas.

Para poder comprobar el nivel competencial de este alumnado, se ha realizado un cuestionario ad-hoc utilizando una metodología cuantitativa. Aunque el análisis evaluativo general de los procesos formativos y de los resultados obtenidos se realizará con una combinación mixta de estrategias e instrumentos cualitativos (análisis de productos, entrevistas, grupos focales, etc.) y cuantitativos (cuestionario y escalas), este trabajo se focaliza en el análisis de la aplicación pretest y el postest del cuestionario ad-hoc de la experiencia del alumnado. El cuestionario se diseña específicamente dentro del programa de $\mathrm{I}+\mathrm{D}+\mathrm{i}$ evaluándose por jueces externos expertos en la temática. El instrumento se compone de 10 variables descriptivas iniciales referentes al sexo, Grado de Magisterio, Universidad, estudios anteriores en centro público, privado o concertado, estudios de los progenitores, estudios de magisterio anteriores, recursos TIC de uso personal, académico y profesional, aprendizaje de TIC en Secundaria y en el entorno familiar y acceso a Internet privado.

Se distribuye un conjunto de 49 ítems sobre dos aspectos: el primero de ellos se refiere a si la redacción del aspecto planteado realmente valora lo que se quiere medir (la columna para valorar este aspecto es la (1); el segundo aspecto a valorar es si la redacción de la cuestión formulada es adecuada para estudiantes de primero de carrera. La valoración de este segundo aspecto se realizará en la columna (2). Todo ello, siguiendo el Marco Común de Competencia Digital Docente (INTEF, 2017), en torno a las áreas de: acceso a la información, comunicación, creación de contenidos, seguridad y resolución de problemas. Aplicándose una escala tipo Likert del 1 al 5, donde 1 es muy desfavorable y 5 significa muy favorable.

\section{Análisis, resultados y discusión}

Se ha aplicado la técnica multivariante de análisis factorial para reducir, estandarizar y validar la información recogida en la escala aplicada. El análisis factorial se ha realizado mediante el procedimiento de componentes principales $(\mathrm{PrC})$ con fines exploratorios. Inicialmente, la determinación del número de factores se ha elaborado según el criterio de 
raíz latente de Kaiser-Meyer-Olkin y, posteriormente, se ha aplicado la rotación varimax (máxima varianza) con normalización de Kaiser, tal y como muestra tabla n. ${ }^{\circ} 1$. La prueba de esfericidad de Bartlett confirma la existencia de factores subyacentes en la matriz de datos por su alto nivel de significación obtenido. El análisis se ha realizado seleccionando un índice de discriminación de 0,5, para realizar la validez de constructo de los 51 ítems.

Tabla 1

Resumen estadístico del análisis factorial

\begin{tabular}{lll}
\hline Medida de adecuación muestral & Kaiser.Meyer-Olkin & 0,867 \\
\hline Prueba de esfericidad de Bartlett & Chi- cuadrado & 7260,477 \\
\cline { 2 - 3 } & Gl. & 1275 \\
\cline { 2 - 3 } & Sig. & 0,000 \\
\hline
\end{tabular}

Fuente: Elaboración Propia.

La solución definitiva del análisis factorial define tres factores que integran 19 de los ítems originalmente propuestos. La tabla $\mathrm{n}^{\circ} 2$ recoge el análisis de esos factores explicativos del 31,268\% de la varianza, con un índice mínimo de discriminación de 0,5.

Tabla 2

Varianza explicada y agrupación de los ítems en factores (rotación varimax)

\begin{tabular}{lll}
\hline Factores & $\begin{array}{l}\text { Varianza explica- } \\
\text { da por factor }\end{array}$ & Ítem que integran cada factor \\
\hline F-1: & $16,978 \%$ & $1,10,37,38,44,45,47$, \\
\hline F-2: & $5,508 \%$ & $14,21,22,23,33,34,35$ \\
\hline F-3: & $5,031 \%$ & $27,29,31,32$ \\
\hline F-4 & 3,799 & $12,13,24$ \\
\hline Varianza total explicada & $31,316 \%$ & \\
\hline Número de factores & 4 & \\
\hline
\end{tabular}

Fuente: Elaboración Propia.

Se ha determinado la estabilidad de la escala cuando es aplicada a una misma persona en diferentes ocasiones y condiciones similares. El Alfa de Cronbach respecto a la totalidad de la escala es de 0,786. La eliminación de cada ítem respecto a la totalidad, aporta los siguientes datos (ítem $n^{\circ} 33=0,770<0,789=$ ítem n. ${ }^{\circ} 22$ ). De manera que no se suprime ningún ítem, ya que al eliminar respectivamente cada uno de 
los ítems, el resultado no aporta un alfa superior al de la totalidad de la escala.

Del mismo modo, se aplicó el Alfa de las dos mitades para conocer el nivel de consistencia entre las dos partes del cuestionario. En los ítems impares se obtuvo un alfa de 0,695, mientras que en los ítems pares un alfa de 0,710 . Por consiguiente, hay una consistencia razonable en la fiabilidad de los ítems pares respecto a los impares.

De manera general, el análisis de las medidas de tendencia central y dispersión pone de manifiesto que los alumnos entrevistados presentan dificultades o desconocimiento (ya que "casi nunca" realizan esas acciones) en torno a los siguientes ítems:

- Ítem 21. Consultas las disposiciones legales que regulan los derechos y deberes de quienes emitimos y recibimos mensajes digitales.

- Ítem 33. Te preocupas de conocer las normativas que regulan los derechos de autor según el tipo de licencia para protegerlos.

- Ítem 34. Respetas las obligaciones y derechos previstos en la normativa que regulan las licencias de uso de los materiales creados por los autores.

- Ítem 35. Te preocupas de conocer los procedimientos por los cuales un autor puede liberalizar sus derechos para hacer su obra de dominio público y gratuito.

- Ítem 27. Gestionas espacios web donde publicar contenidos multimedia publicados por ti.

- Ítem 29. Creas audios digitales mezclando voz y música.

La desviación típica de los ítems reseñados pone de manifiesto que la dispersión de las puntuaciones es media, ya que la sigma oscila entre Ítem $n^{\circ} 44=0,263<1,584=$ Ítem 35, con una máxima desviación típica de $\alpha=2,5$.

El resto de ítems cuya media es mayor, comprendidas entre Ítem 32 $\left(M=2,74<3,47\right.$, Ítems $\left.n^{\circ} 32\right)$ evidencia la realización de esas acciones con cierta frecuencia, pero no de modo continuado. Consiguientemente, los ítems comprendidos en ese intervalo también necesitan apoyo para su desempeño más frecuente, pero en menor medida que los anteriores.

Con todo, se presenta a continuación el análisis pormenorizado de los ítems reseñados dentro de las diferentes dimensiones desde el estudio de las medidas de tendencia central en el pretest y el postest realizado. 
La dimensión I (tabla 3) incluye el análisis de todos aquellos aspectos relacionados con la autonomía de la persona respecto a la navegación en red y la resolución de problemas técnicos sobrevenidos (identificación, resolución y búsqueda y consulta de soluciones). Todas las medias analizadas aumentan en el estudio de postest, observándose un aumento significativo en el ítem $n^{\circ} 1$ (dif $>0,5$ ). A pesar de que esta dimensión parte de unas puntuaciones de pretest bastante positivas, los resultados de postest muestran que han sido perfeccionadas. Lo que pone de manifiesto una mejor habilidad en torno a la configuración del navegador web y el uso de espacios de almacenamiento tras la aplicación del programa formativo. Así como una mayor autonomía ante la necesidad de identificar, buscar y resolver problemas técnicos.

Tabla 3

Medias y desviaciones típicas del pretest y el postest de la Dimensión I. Navegación y resolución de problemas técnicos

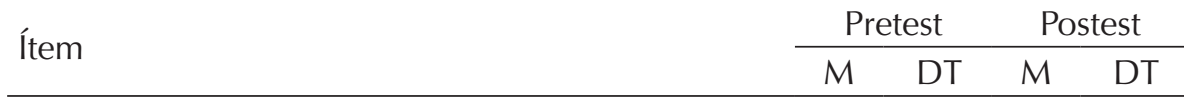

1. Configuras el navegador web de tu ordenador

(Chrome, Mozilla, Explorer...,) cuando lo conside- 3,04 1,371 3,66 1,446 ras necesario.

10. Utilizas espacios de almacenamiento externo con ese fin (discos duros, nubes y memorias USB).

$3,72 \quad 1,328 \quad 4,16 \quad 1,402$

37. Realizas operaciones básicas de mantenimiento

y protección de los dispositivos que utilizas: actua-

lizaciones de sistemas, actualizaciones de acceso, 3,88 1,118 4,05 1,117

antivirus, contraseñas de acceso, etc.

38. Sabes cómo actuar cuando detectas una amenaza de seguridad en tus dispositivos y equipos.

$3,41 \quad 1,258 \quad 3,72 \quad 1,388$

44. ¿Identificas un problema técnico explicando con claridad en qué consiste el mal funcionamiento?

$2,92 \quad 1,263 \quad 3,36 \quad 1,384$

45. Resuelves problemas técnicos no complejos relacionados con dispositivos y entornos digitales habituales con la ayuda de un manual o información técnica disponible.

47. Consultas foros especializados que te ayuden a resolver dudas o problemas

$3,37 \quad 1,133 \quad 3,73 \quad 1,051$ Fuente: Elaboración Propia.

La dimensión II (tabla 4) se refiere al conocimiento y cumplimiento de la normativa vigente relacionada con las disposiciones legales, los 
Efectos del programa affective e-learning en el desarrollo de la Competencia Digital en estudiantes del Grado en Educación Primaria

Álvaro Pérez García y Alba María Hernández-Sánchez

derechos de autor y las licencias para proteger el material en línea. De manera general, el grupo de participantes muestra una baja tendencia al conocimiento y cumplimiento de los aspectos incluidos. Sin embargo, la mayoría de los ítems puntúan de forma más alta tras haberse realizado la formación específica, destacándose los ítems n $\mathrm{n}^{\circ} 14$ y 22 con un cambio significativamente positivo (dif $>0,5$ ). No obstante, existen dos ítems que han obtenido una media ligeramente más baja en los resultados recogidos en el postest (ítems $\mathrm{n}^{\circ} 23$ y 35), ambos referentes a cuestiones actitudinales de preocupación por conocer procedimientos para liberalizar derechos de autor y de abordar un intercambio comunicativo en línea teniendo en cuenta las características socioculturales de la audiencia.

Tabla 4

Medias y desviaciones típicas del pretest y el postest de la Dimensión II. Disposiciones legales, derechos de autor y licencias

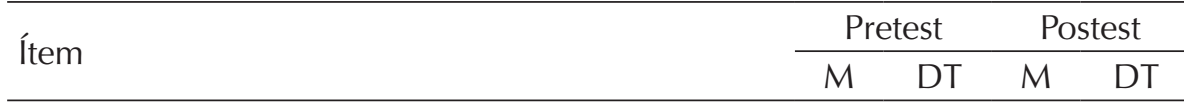

14. Cuando compartes información o contenidos obtenidos en líneas, citas el autor y la fuente, si- 3,13 1,251 3,71 1,093 guiendo las normas existentes

21. Consultas las disposiciones legales que regulan los derechos y deberes de quienes emitimos y reci- 2,40 $1,386 \quad 2,64 \quad 1,398$ bimos mensajes digitales

22. Cuando emites y recibes mensajes digitales cumples las normas legales que regulan la transfe- 3,72 1,977 4,32 1,203 rencia de información

23. Tienes presentes las características socioculturales de las audiencias hacia las que diriges tus mensajes digitales que crean para difundirlos a través $3,47 \quad 1,453 \quad 3,38 \quad 1,226$ de internet

33. Te preocupas de conocer las normativas que regulan los derechos de autor según el tipo de licen- 2,51 1,353 2,66 1,252 cia para protegerlos

34. Respetas las obligaciones y derechos previstos en la normativa que regulan las licencias de uso de 3,37 1,543 3,68 1,262 los materiales creados por los autores

35. Te preocupas de conocer los procedimientos por

los cuales un autor puede liberalizar sus derechos 2,64 1,584 2,60 1,245 para hacer su obra de dominio público y gratuito Fuente: Elaboración Propia. 
Por su parte, la dimensión III (tabla 5) sobre "desarrollo, integración y estructuración de contenidos" del cuestionario aplicado en el estudio se focaliza en el desarrollo de las habilidades que involucran una mayor implicación de la persona en términos de creación (producción, reelaboración y formación de productos genuinos), evaluación (aplicación de pensamiento crítico en la gestión y la generación) y análisis (relación e interrelación en la edición y reproducción). Observándose en el pretest del análisis de las medidas de tendencia central de la dimensión una baja competencia del alumnado en el desarrollo de habilidades de pensamiento de nivel superior que implican la gestión de espacios web, la creación de audios y vídeos digitales y la manipulación de programas de edición.

En todos los ítems analizados se observa una leve mejoría en el manejo de los aspectos reseñados. Sin embargo, en ningún caso se puede afirmar que haya un cambio amplio, hipotéticamente inducido por la necesidad de una visión más longitudinal para la consecución de una mejora significativa que se requiere en aprendizajes tan procedimentales como los que aglutina esta dimensión.

Tabla 5

Medias y desviaciones típicas del pretest y el postest de la Dimensión III. Desarrollo, integración y estructuración de contenidos

\begin{tabular}{lllll}
\hline Ítem & \multicolumn{3}{l}{ Pretest } & \multicolumn{2}{l}{ Postest } \\
\cline { 2 - 5 } & M & DT & M & DT \\
\hline $\begin{array}{l}\text { 27. Gestionas espacios web donde publicar conte- } \\
\text { nidos multimedia publicados por ti }\end{array}$ & 2,52 & 1,318 & 2,74 & 1,321 \\
\hline $\begin{array}{l}\text { 29. Creas audios digitales mezclando voz y música. } \\
\text { 21. Creas videos propios insertando fotografías, }\end{array}$ & 1,233 & 2,64 & 1,354 \\
\hline $\begin{array}{l}\text { 3ráficos, fragmentos de películas y textos } \\
\text { gran }\end{array}$ & 1,25 & 3,27 & 1,240 \\
\hline
\end{tabular}

32. Usas programas de edición digital (de audio

y/o video) para modificar contenidos creados por ti $2,75 \quad 1,811 \quad 2,89 \quad 1,216$ o por otros autores.

Fuente: Elaboración Propia.

Finalmente, la dimensión IV (tabla 6) refleja cuáles son las competencias digitales que más presencia tienen en la vida cotidiana del estudiantado universitario actual, obteniéndose una puntuación muy positiva incluso en el pretest del cuestionario. De modo que la interacción a través de los medios digitales y la gestión de la identidad digital se 
Efectos del programa affective e-learning en el desarrollo de la Competencia Digital en estudiantes del Grado en Educación Primaria

Álvaro Pérez García y Alba María Hernández-Sánchez

pueden señalar como las competencias más desarrolladas del grupo de participantes.

Igualmente, dos de los tres ítems reseñados han visto mejorada su puntuación media en el estudio de postest (ítems $n^{0} 13$ y 24), referentes a la interacción con fuentes diversas de información y a la gestión de la identidad personal digital, mientras que el uso de herramientas digitales para interactuar con los demás se ve levemente disminuido respecto a la evaluación de pretest. Pese a no tener datos que confirmen este hecho, se plantea la necesidad de estudiar variables extrañas que puedan incidir en este cambio, como el momento de desarrollo del curso coincidente con un momento inicial de mayor disponibilidad horario y un momento final donde se limita la disponibilidad de tiempo.

Tabla 6

Medias y desviaciones típicas del pretest y el postest de la Dimensión IV. Interacción a través de medios digitales y gestión de la identidad digital

\begin{tabular}{lllll}
\hline & \multicolumn{2}{l}{ Pretest } & \multicolumn{2}{l}{ Postest } \\
\cline { 2 - 5 } & $M$ & DT & $M$ & DT \\
\hline $\begin{array}{l}\text { 12. Utilizas, al menos, tres herramientas digitales } \\
\text { distintas para interactuar con los demás (correo } \\
\text { electrónico, Whatsapp, chat de texto, video-chat, } \\
\text { redes sociales, etc.) }\end{array}$ & 4,73 &,- 687 & 4,45 & 1,154 \\
\hline $\begin{array}{l}\text { 13. Cuando navegas, interacciones con diferentes } \\
\text { fuentes de información digital (imágenes, enlaces, } \\
\text { audios, videos, repositorios de descarga) }\end{array}$ & $4,23,9067$ & 4,52 & 1,208 \\
\hline $\begin{array}{l}\text { 24. Gestionas tu identidad digital, cuidando de } \\
\text { hacer públicos solo aquellos datos personales que } \\
\text { deseas que se conozcan. }\end{array}$ & $4,41,955$ & 4,67 & 1,371 \\
\hline
\end{tabular}

Fuente: Elaboración Propia.

\section{Conclusiones}

Teniendo en consideración los resultados obtenidos en el pretest, en el que se reflejaba las carencias de diversos aspectos dentro de la Competencia Digital del Marco Común que propone el INTEF, y el programa formativo aplicado para paliar esas limitaciones y dificultades, se puede concluir que la aplicación de este programa basado en un modelo de affective e-learning ofrece unos resultados positivos en la mejora de la competencia digital del alumnado de magisterio de Educación Primaria, 
de manera que se adapta a los conocimientos previos y los distintos ritmos y estilos de aprendizaje del grupo de participantes.

Aspectos como los derechos de autor, el uso de normas de internet, la interacción con diversas fuentes de internet, la gestión de la identidad digital, o la creación y edición de audios y vídeos, han obtenido una mejora en sus puntuaciones tras la realización del programa formativo.

No obstante, se observan aspectos que no pueden ser valorados de manera trasversal y precisan de la aplicación de un programa y un estudio de carácter longitudinal. La adquisición de la competencia digital requiere de un programa basado en los principios del affective e-learning que se extienda a lo largo de los años de formación del futuro grupo de docentes, siendo así posible observar y analizar el proceso de empoderamiento personal de cada uno de los participantes.

\section{Referencias}

Argente, E.; Vivancos, E.; Alemany, J. y García-Fornes, A. (2017). Educando en privacidad en el uso de las redes sociales. Education in the knowledge society, 18 (2), 107-126. https://doi.org/10.14201/eks2017182107126

Bermejo Fernández-Nieto, J. (2017). Identidad digital. Retos para la función docente. Padres y Maestros, 370, 37-42.

Cáceres Zapatero, M.D.; Brändle Señán, G.; Ruiz San Román, J. A. (2017). Sociabilidad virtual: la interacción social en el ecosistema digital. Historia y Comunicación Social, 22(1), 233-247.

Cantón Mayo, I.; Grande de Prado, M. y Cañón Rodríguez, R. (2016). La competencia digital de los futuros maestros. En D. Cobos Sanchiz, J. Gómez Galán \& E. López Meneses (Coords.), La Educación Superior en el Siglo XXI: nuevas características profesionales y científicas (239-263). UMET Press, Universidad Metropolitana, Sistema Universitario Ana G. Méndez, San Juan, PR.

Centeno Moreno, G. y Cubo Delgado, S. (2013). Evaluación de la competencia digital y las actitudes hacia las TIC del alumnado universitario. Revista de Investigación Educativa, 31 (2), 517-536. http://dx.doi.org/10.6018/rie.31.2.169271

Ezquerra Martínez, A.; Burgos Jiménez, E. y Manso Lorenzo, J. (2016). Estudio comparativo sobre las estrategias desarrolladas por los futuros docentes de Primaria y Secundaria en la elaboración de audiovisuales educativos. Revista Eureka sobre Enseñanza y Divulgación de las Ciencias, 13 (2), 493-504.

Esteve Mon, F. (2015). La competencia digital docente. Análisis de la autopercepción y evaluación del desempeño de los estudiantes universitarios de educación por medio de un entorno 3D. Tesis Doctoral inédita. Universitat Rovira y Virgili.

Espuny Vidal, C.; González Martínez, J. y Gisbert Cervera, M. (2010) ¿Empezamos a navegar? La competencia digital profesional en los futuros maestros. En J.M. Pérez 
Efectos del programa affective e-learning en el desarrollo de la Competencia Digital en estudiantes del Grado en Educación Primaria

Álvaro Pérez García y Alba María Hernández-Sánchez

Tornero; J. Cabero Almenara y L. Vílchez (coords.), Alfabetización Mediática y Culturas digitales. Sevilla: Universidad de Sevilla.

Fernández Roldán, A.D. y García García, F. (2016). La interacción digital como dinámica de construcción colectiva desde distintos perfiles de usuarios para el buen trabajo. etic@net, 16 (1),27-38.

Gallego Ortega, J. L.; García Guzmán, A. y Rodríguez Fuentes, A. (2015). ¿Qué ocurre en la mente del estudiante universitario cuando escribe? Estudio de caso con alumnado de Magisterio. Lengua y Habla, 19, 1-20.

García Sempere, P. J. (2014). Vídeo en la educación: creación de subtítulos para romper barreras de accesibilidad. International Journal of Educational Research and Innovation (IJERI), 2, 107-117.

Giones-Valls, A. y Serrat-Brustenga, M. (2010). La gestión de la identidad digital: una nueva habilidad informacional y digital. BiD, 24. DOI: http://dx.doi. org/10.1344/105.000001544

Girón-Escudero, V.; Cózar-Gutiérrez, R. \& González-Calero Somoza, J.A. (2019). Análisis de la autopercepción sobre el nivel de competencia digital docente en la formación inicial de maestros/as. Revista Electrónica Interuniversitaria de Formación del Profesorado, 22(3), 193-218.

Gisbert Cervera, M. y Esteve Mon, F. (2011). Digital Leaners: la competencia digital de los estudiantes universitarios. La Cuestión Universitaria, 7, 48-59.

Hernández-Sánchez, A. M. y Ortega-Maldonado, A. (2015). Hacia la personalización del e-Learning: la afectividad y su repercusión en el bienestar subjetivo. Revista LasaIlista de Investigación, 12(2), 194-203. http://dx.doi.org/10.22507/rli.v12n2a20

Hidalgo Navarrete, J. y Aliaga Zegarra, E. (2020). Análisis de las estrategias didácticas para el diseño, selección, producción, utilización y validación de recursos educativos audiovisuales interactivos en una institución educativa. Estudio inicial. REID, 23, 79-98.

INTEF (2017). Marco Común de Competencia Digital Docente. Madrid: Ministerio de Educación, Cultura y Deporte.

León, L. (2018). Niños YouTubers y el proceso de creación de videos: evidencia de competencias transmedia en acción. Comunicación y Sociedad, 33, 115-137. https://doi. org/10.32870/cys.v0i33.7080

Marín Díaz, V. (2016). El blog: pensamiento de los profesores en formación en Educación Primaria. Opción, Año 32, 79, 145 - 162.

Mayorga Fernández, M.J.; Madrid Vivar, D. y Núñez Avilés, F. (2011). La competencia digital de los docentes: formación y actualización en web 2.0. etic@net, 11, 213232.

Molina Jaén, M.D., Pérez García, A. y Antiñolo Piñar, J.L. (2012). Las TIC en la formación inicial y en la formación permanente del profesorado de infantil y primaria. EDUTEC, Revista Electrónica de Tecnología Educativa, 41.

Ortega Carrillo, J.A. y Pérez García, A. (2013). El cine digital en la formación inicial del profesorado: una experiencia innovadora realizada en la Universidad de Granada. Educación XXI, 16 (2), 297-320. DOI: 10.5944/educxx1.16.2.2644

Portillo Fernández, J. (2016). Planos de realidad, identidad virtual y discurso en las re- 
Efectos del programa affective e-learning en el desarrollo de la Competencia Digital en estudiantes del Grado en Educación Primaria Álvaro Pérez García y Alba María Hernández-Sánchez

des sociales. Logos: Revista de Lingüística, Filosofía y Literatura, 26(1), 51-63. DOI: dx.doi.org/10.15443/RL2604

Raro López (2012). Los textos expandidos. La forma de la escritura tecnológica: un análisis pragmaestilístico del entorno comunicativo de los blogs. Tesis Doctoral Inédita. Universitat Jaume I.

Rodríguez-López, J. (2017). El videoclip interactivo. Nuevos modelos de alfabetización mediática en adolescentes. Revista Mediterránea de Comunicación/Mediterranean Journal of Communication, 8(2), 161-167. https://www.doi.org/10.14198/MEDCOM2017.8.2.11

Roldán, P. y Sabulsky, G. (2012). Futuros docentes en tiempos de convergencia digital. $V E s C, 4,11-25$.

Romero Martínez, S.J.; Hernández Lorenzo, C.J. y Ordóñez Camacho, X.G. (2016). La competencia digital de los docentes en educación primaria: análisis cuantitativo de su competencia, uso y actitud hacia las nuevas tecnologías en la práctica docente. $C E F, 4,33-51$.

Serrano-Puche, J. (2013). Vidas conectadas: tecnología digital, interacción social e identidad. Historia y Comunicación Social, 18 ( $\mathrm{N}^{\circ}$ Esp.), 353-364. http://dx.doi. org/10.5209/rev_HICS.2013.v18.44249

Torres Climent, Á.L. (2009). Creación y utilización de vídeo digital y TICS en Física y Química. Revista Eureka sobre Enseñanza y Divulgación de las Ciencias, 6 (3), 440-451.

Vázquez-Cano, E.; Reyes Vélez, M.; Colmenares Zamora, L. y López Meneses, E. (2017). Competencia digital del alumnado de la Universidad Católica de Santiago de Guayaquil. Opción, Año 33, 83, 229-251. 
